\title{
Estudio de la interacción creep - fatiga en aceros ferríticos
}

\section{Study of creep - fatigue interaction in ferritic steels}

Presentación: 6/10/2020

\section{Doctorando:}

\section{Alejandro STAFFA}

Grupo de Estudio sobre Materiales, Facultad Regional Bahía Blanca, Universidad Tecnológica Nacional - Argentina

astaffa@frbb.utn.edu.ar

\section{Director/a:}

\section{Lilian Moro}

\section{Co-director/a:}

\section{Lucio Iurman}

\section{Resumen}

El fenómeno de interacción creep-fatiga se presenta cuando el material se somete a temperaturas suficientemente altas y ciclos de tensiones que no solo producen deformaciones de creep, sino también deformaciones por fatiga. Esta combinación de efectos reduce seriamente la vida de los componentes, razón por la cual, resulta de interés industrial.

Se estudió bajo estas solicitaciones al acero de baja aleación $1 \mathrm{Cr}$ 0,5Mo realizando ensayos cortos a $600{ }^{\circ} \mathrm{C}, 168 \mathrm{MPa}$, 131 MPa y 30 minutos de permanencia en los picos de tensión, que permitieron guiar la investigación hacia ensayos que simulen condiciones industriales. Posteriormente, se analizó el comportamiento del acero cuando se somete a la interacción de creep-fatiga, con cargas cíclicas entre 20 y $131 \mathrm{MPa}$ sin permanencia con duraciones de 10 y 1342 ciclos a igual frecuencia, seguido de un régimen estacionario de creep a esfuerzos contantes a la tensión y temperatura de diseño (70 MPa y $540{ }^{\circ} \mathrm{C}$ ). Los resultados se compararon en todos los casos con los obtenidos al estudiar el material solo sometido a la condición de creep. Este tipo de solicitación termo-mecánica, se presenta en sobrecargas aisladas en plantas de proceso continuo de la industria del petróleo y gas, petroquímica y de generación de energía, pudiendo dar lugar a fallas tempranas del material.

Dado que se ensayaron probetas a esfuerzos de fatiga con un valor de tensión media positiva, el material presentó el efecto ratcheting y como consecuencia del mismo, una acumulación de deformaciones plásticas que producen la degradación del acero y la disminución de su resistencia al creep.

Palabras clave: Creep-fatiga, aceros ferriticos, ratcheting, altas temperaturas, resitencia al creep

\section{Abstract}

The creep-fatigue interaction phenomenon occurs when the material is subjected to sufficiently high temperatures and stress cycles that not only produce creep deformations, but also fatigue deformations. This combination of effects seriously reduces the life of the components, which is why it is of industrial interest. Under these requests, $1 \mathrm{Cr} 0.5 \mathrm{Mo}$ low alloy steel was studied under these requests by conducting short tests at $600{ }^{\circ} \mathrm{C}, 168 \mathrm{MPa}, 131 \mathrm{MPa}$ and 30 minutes of hold periods at peaks, which led the research to tests that simulate industrial conditions. Subsequently, the behavior of steel was analyzed when subjected to the interaction of creep-fatigue, with cyclic loads between 20 and $131 \mathrm{MPa}$ without permanence with durations of 10 and 1342 
cycles at equal frequency, followed by a stationary regimen of creep at stress-counting stress and design temperature (70 MPa and $540^{\circ} \mathrm{C}$ ). The results were compared in all cases with those obtained by studying the material only subject to the creep condition. This type of thermo-mechanical request is presented in isolated overloads in continuous process plants of the oil and gas industry, petrochemical and power generation, which can lead to early material failures.

Since tested specimens at fatigue stresses with a positive mean stress value, the material presented the ratcheting effect and as a result of it, an accumulation of plastic deformations that cause the degradation of steel and the decrease of its resistance to creep.

Keywords: Creep-fatige, ferritic Steel, ratcheting, creep strength, heat temperature

\section{Introducción}

La interacción creep - fatiga se presenta en componentes de plantas de potencia que operan a alta temperatura y sometidos a estados cíclicos de tensión. Este fenómeno depende del grado y velocidad de deformación, de la duración de los ciclos de tensión, y de la resistencia y ductilidad del material (Viswanathan, 1989) (Halford, 1997).

Cuando el material presenta deformaciones por creep provocadas por la temperatura de servicio y en forma simultánea se somete a estados cíclicos de tensiones (fatiga), sufre una disminución de su vida útil. Ambos efectos, que actúan uno sobre el otro, deben ser cuantificados para lograr mejorar el rendimiento de los componentes. El objetivo del presente trabajo es el estudio de la variación de la resistencia mecánica del acero ferrítico $1 \mathrm{Cr}$ 0.5Mo cuando está sometido a la interacción creep fatiga y comparar con el comportamiento del material cuando solo está sometido a creep (Holdsworth, 2011) (Zhang \& Jarir, 2018).

Las técnicas experimentales utilizadas se encuentran normalizadas (ASTM E139-11, 2011) (ASTM E2714-13, 2013). Se usó un equipamiento desarrollado por el Grupo de Estudios en Materiales, de ensayo múltiple, con variables controladas de temperatura y tensión, velocidades de carga y descarga, tiempos de permanencia y variables medidas de deformación, tiempo y ciclos (Molina, Pender, Gonzalez, \& Moro, 2018).

\section{Desarrollo}

El material bajo estudio es un acero ferrítico de baja aleación, para uso en cañerías a alta temperatura, de especificación SA 335 Gr P11, el cual presenta alta resistencia al creep y bajo coeficiente de expansión térmica. En primer lugar, se determinó la composición química del material mediante un espectrómetro de emisión por plasma, marca Spectromax modelo X. En la Tabla 1, se presentan los elementos componentes y los valores medidos de las concentraciones (\% en peso) del material. El material se ensaya en condición de suministro, la cual consta de tratamientos térmicos de normalizado y revenido a $650^{\circ} \mathrm{C}$, que generan una micro-estructura de matriz ferrítica con precipitados estables de carburos y nitruros.

\begin{tabular}{|c|c|c|c|c|c|c|c|c|c|c|}
\hline Material & $\mathbf{C}$ & $\mathbf{C r}$ & Mo & $\mathbf{V}$ & $\mathbf{N i}$ & $\mathbf{M n}$ & $\mathbf{S i}$ & $\mathbf{C u}$ & Otros & $\mathbf{F e}$ \\
\hline $\begin{array}{c}\text { SA 335 } \\
\text { Grado P11 }\end{array}$ & 0.06 & 1.01 & 0.46 & -- & 0.10 & 0.43 & 0.85 & 0.11 & $\mathrm{~S}, \mathrm{Sn}<0.011$ & Balance \\
\hline
\end{tabular}

Tabla 1: Composición química del acero $1 \mathrm{Cr}-0.5$ Mo.

Se caracterizó el comportamiento del material sometido a tracción estática a una temperatura de $600{ }^{\circ} \mathrm{C}$. Se obtuvieron los siguientes valores: resistencia máxima de $257 \mathrm{MPa}$, límite elástico $(0,15 \%) 168 \mathrm{MPa}$, deformación total del 15 \% y módulo de elasticidad de 58 GPa. La simulación de la interacción creep - fatiga, se logró utilizando el equipo de creep por tracción y provocando sobre la probeta, ciclos de carga y descarga, siendo el sentido siempre positivo. Para provocar fatiga de bajo número de ciclos, se decide aplicar una tensión máxima igual al límite elástico, $168 \mathrm{MPa}$, y una tensión mínima de $20 \mathrm{MPa}$, figuras 1 y 2. 
Previamente, se realizó un ensayo de creep a $600{ }^{\circ} \mathrm{C}$ y $168 \mathrm{MPa}$, arrojando como resultado una duración de 80 hs y una deformación máxima de 12,5 \%. Para determinar el grado de afectación propia del fenómeno de fatiga mecánica sobre el creep, se realizaron ensayos con una velocidad de carga y descarga de $30 \mathrm{MPa} /$ minuto y se ensayaron probetas con ciclos sin permanencia y con permanencia de 30 minutos en el pico de tensión máxima. En la Tabla 2 se muestra un estudio comparativo del ensayo de creep y del que indica el fenómeno creep - fatiga, ambos estudios se realizaron a la misma temperatura.

\begin{tabular}{|c|c|c|c|c|}
\hline \multicolumn{2}{|c|}{} & \multirow{2}{*}{ Creep } & \multicolumn{2}{c|}{ Creep - Fatiga } \\
\cline { 4 - 5 } & & Sin permanencia & Con permanencia \\
\hline Duración & hs & 80 & 3 & 7 \\
\hline $\mathrm{N}^{\circ}$ de ciclos & $\mathrm{N}$ & --- & 22 & 15 \\
\hline Deformación & $\%$ & 12,5 & 0,90 & 11,27 \\
\hline
\end{tabular}

Tabla 2: Resultados comparativos a $600^{\circ} \mathrm{C}$ ensayos de creep $168 \mathrm{MPa}$, creep fatiga 20/168 MPa sin y con permanencia de $30^{\prime}$.

En la figura 1 y 2 se muestran las deformaciones en función del tiempo de los ensayos sin permanencia y con una permanencia de treinta minutos respectivamente, puede observarse que en el primer caso las deformaciones son crecientes a igual tensión, por lo tanto, el material ablanda con los ciclos. El ablandamiento por fatiga podría explicarse por la generación de defectos puntuales que deja parcialmente el metal, producto de la activación térmica, permitiendo el trepado de dislocaciones fuera del plano de deslizamiento (Dieter, 1988). La ausencia de período terciario de Creep, sumado a la inexistencia de fisuras (Figura 3), nos permite concluir en este caso que predomina la fatiga sobre el creep, dando lugar al fenómeno de ratcheting. Otro aspecto observable es que como consecuencia de la baja velocidad de carga y descarga, los picos de tensión y deformación no coinciden temporalmente, esto se debe a que la velocidad de deformación es mayor que la velocidad de descarga. También y por el mismo motivo, se observa que el material presenta expansión adiabática en los ciclos de carga y descarga.

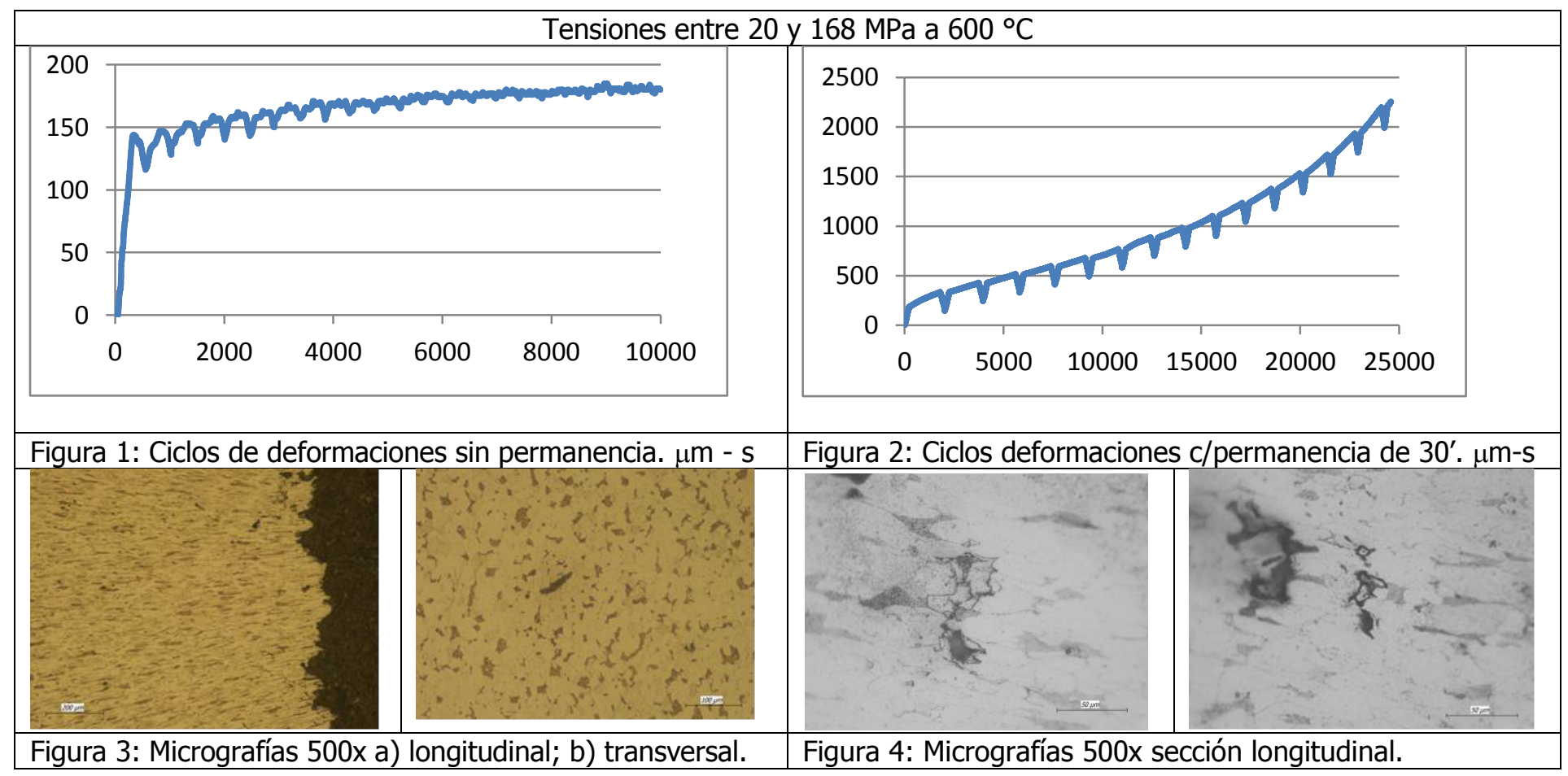

En la Figura 3 a), pueden observarse granos alargados en la dirección de deformación, no se encuentran fisuras. Se advierte la presencia de huecos aislados. En la micrografía b), se pueden observar granos equiaxiales de ferrita y un disperso de bainita parcialmente globulizada. En la figura 4, se pueden observar micrografías donde se aprecian microfisuras intergranulares, tanto en la interfaz Bainita- Ferrita como en el borde de granos vecinos de la Ferrita. La fisuración intergranular es característica de creep, debido a que la resistencia mecánica del borde es inferior a la del 
interior del grano, contrariamente a lo que ocurre a menores temperaturas. La deformación entonces, tiene lugar en los bordes de grano. Durante esta deformación, se producen descohesiones locales, fisuras intergranulares y formación de huecos (Dieter, 1988). A partir de los resultados indicados en la figura 2 y la tabla 2, se observa la disminución de la vida útil del material por los ciclos de carga y reducción de la rigidez del material, provocada por la presencia de fisuras y cavidades y un aumento de la velocidad de deformación a medida que avanza el ensayo, esta es una característica del período terciario de creep. Se concluye que en este caso predomina el creep.

Se observa, tanto en el ensayo sin permanencia como en el ensayo con permanencia, que los ciclos de carga aplicados producen ciclos de deformación entre 0,92 a 1,08 \%, sensiblemente superior a lo indicado en trabajos de investigación, que oscilan en 0,25 a 0,75\%. Esta última observación, se tomó en cuenta para el segundo grupo de ensayos que se indican a continuación.

A partir de las observaciones y conclusiones extraídas del primer grupo de ensayos, se decidió realizar ensayos con y sin permanencia, a la misma temperatura, $600{ }^{\circ} \mathrm{C}$, pero bajando los ciclos de tensiones entre 20 y $131 \mathrm{MPa}$, persiguiendo dos objetivos: bajar la amplitud de deformaciones y aumentar el número de ciclos de vida del material. Se observa que disminuyeron las deformaciones plásticas por ciclo, las mismas oscilaron el 0,12 a 0,38 \%. En la tabla 3 se indican los resultados comparativos del ensayo de creep y de los de creep - fatiga sin y con permanencia de treinta minutos.

\begin{tabular}{|c|c|c|c|c|}
\hline \multicolumn{2}{|c|}{} & \multirow{2}{*}{ Creep } & \multicolumn{2}{c|}{ Creep - Fatiga } \\
\cline { 4 - 5 } & & Sin permanencia & Con permanencia \\
\hline Duración & hs & 554 & 9 & 9 \\
\hline $\mathrm{N}^{\circ}$ de ciclos & $\mathrm{N}$ & --- & 126 & 22 \\
\hline$\dot{\varepsilon}$ & $\% / \mathrm{h}$ & 0,00171 & --- & 0,26 \\
\hline Deformación & $\%$ & 11,75 & 0,40 & 4,4 \\
\hline Rotura & & Sí, dúctil tipo copa y cono & No \\
\hline
\end{tabular}

Tabla 3: Datos obtenidos de ensayos a $600^{\circ} \mathrm{C}$; Creep a $131 \mathrm{MPa}$ y creep fatiga entre 20 y $131 \mathrm{MPa}$.

En la figura 5 se muestran las curvas obtenidas con los resultados de tres ensayos realizados a $600{ }^{\circ} \mathrm{C}$. Previamente se realizó un ensayo de creep a $131 \mathrm{Mpa}$. El identificado con la línea C azul, es una parte del ensayo de creep a $131 \mathrm{MPa}$; el correspondiente a la línea verde es el ensayo de creep-fatiga con ciclos de 20 a 131 MPa sin permanencia (C-F s/p) y finalmente, el ensayo identificado con la línea roja ( $\mathrm{C}-\mathrm{F} \mathrm{c} / \mathrm{p}$ ) posee los mismos parámetros de tensión que el anterior pero una permanencia en los picos de tensión de 30 minutos.

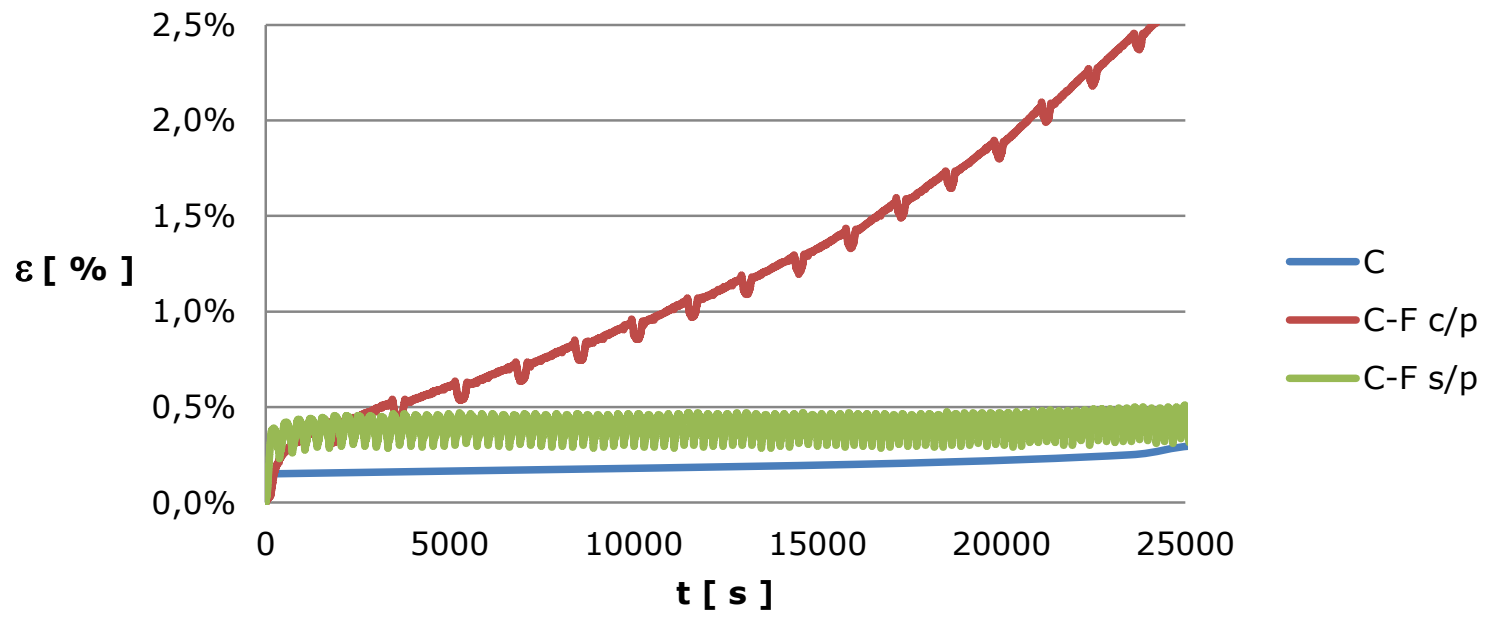

Figura 5: Ensayos de Creep y Creep-Fatiga a $131 \mathrm{MPa}$ (máxima) y $600{ }^{\circ} \mathrm{C}$ 
Se observa, que la condición más severa ha sido la de creep-fatiga sin permanencia dado que alcanzo la rotura a las 9 hs. Sin embargo, comparando con los resultados de la figura 1 se advierte que en el caso del creep-fatiga con permanencia, al cabo de igual tiempo $(9 \mathrm{hs})$ presenta una velocidad en aumento que predice su rotura (se aprecia el ingreso al periodo terciario).

Se desprende de la tabla 1 y de la figura 1, un incremento muy significativo de la velocidad mínima de creep-fatiga respecto de la velocidad mínima de creep, que confirma la reducción de vida útil teniendo en cuenta que el material no deformará más de un $15 \%$. Puede observarse en el ensayo de creep sin permanencía, las deformaciones son crecientes a igual tensión, por lo tanto, el material repite la característica de ablandamiento con los ciclos. También como carece del periodo terciario característico de creep, se concluye que predomina el fenómeno de fatiga. Se presenta también el efecto ratcheting.

En la figura 6, se muestra la estructura de la superficie externa, donde se observa la presencia de microfisuras paralelas al eje longitudinal de la probeta, algunas de las cuales progresan a través de las cavidades de creep. Esto resulta consistente, ya que la fisuración intergranular es característica en creep y se produce porque la resistencia mecánica del borde es inferior respecto de la del interior del grano y en este caso, los bordes más deformados están en la dirección longitudinal. Contrariamente, si las fisuras se debieran a la fatiga, se orientarían preferentemente perpendiculares al esfuerzo. Se concluye en este caso, que el daño predominante corresponde a mecanismos de creep.

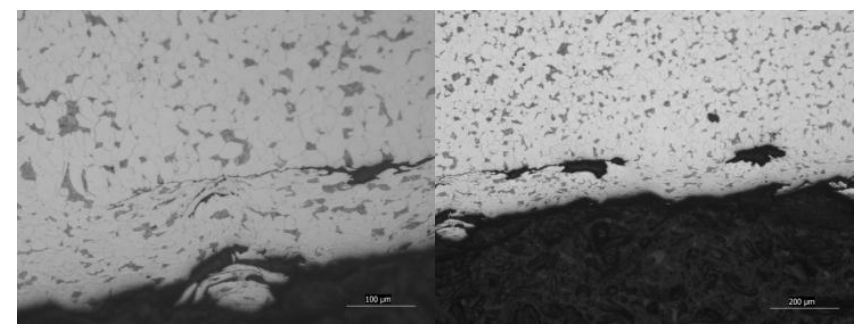
a) $200 x$
b) $100 x$

Figura 6: Micrografías realizadas en la muestra sometida a ciclos con permanencia y una tensión de $131 \mathrm{MPa}$.

Por último se realizaron ensayos de creep y de creep-fatiga a $540{ }^{\circ} \mathrm{C}$, para lo cual primero se sometió el material a cargas cíclicas y luego se aplicó una tensión constante experimentando el fenómeno de creep. La fatiga del material se experimentó sometiendo el acero a ciclos de tensión de 20 y 131 MPa y luego se continuó con un esfuerzo constante a 70 $\mathrm{MPa}$ (período de creep). Se efectuaron dos tipos de ensayo, los primeros de bajo ciclos (10 ciclos) y luego aumentando la cantidad de ciclos (1342 ciclos). En la tabla 4 se indican los resultados de los ensayos de creep - fatiga y se compararan con los obtenidos al someter el material a la condición de creep a $70 \mathrm{MPa}$ y $540^{\circ} \mathrm{C}$ (valores de diseño) (Zhang \& Jarir, 2018).

\begin{tabular}{|c|c|c|c|}
\hline Variable & $\begin{array}{c}\text { Ensayo C-F } \\
\text { con 10 ciclos }\end{array}$ & $\begin{array}{c}\text { Ensayo C-F } \\
\text { con 1342 ciclos }\end{array}$ & Ensayo de Creep \\
\hline Deformación primer pico de tensión (\%) & 0,52 & 0,62 & --- \\
\hline Deformación al inicio de la etapa secundaria (\%) & 0,50 & 0,27 & 0,56 \\
\hline Velocidad mínima de creep (\%/h) & 0,0010 & 0,0040 & 0,000041 \\
\hline Duración del ensayo (h) & 74 & 60 & 2313 \\
\hline Deformación Máxima (\%) & 0,63 & 0,38 & 0,66 \\
\hline
\end{tabular}

Tabla 4: Estudio comparativo de los resultados obtenidos de los ensayos de creep - fatiga y creep a $540{ }^{\circ} \mathrm{C}$.

Se trazaron los gráficos deformación tiempo para cada una de las situaciones. En las figuras 7 y 8 se muestra el ensayo de creep - fatiga con 10 ciclos de fatiga, la primera (Fig. 7) muestra la primera etapa, mientras que en la segunda se observa el ensayo completo (Fig. 8). El efecto ratcheting tiene lugar durante los ciclos de creep - fatiga y se produce la acumulación de deformaciones plásticas. En los primeros 3 ciclos se observa un endurecimiento del material, mientras que desde el pico de tensión $4^{\text {to }}$ al $10^{\text {mo }}$ hay un ablandamiento del mismo (Zhang \& Jarir, 2018). El ensayo continua con la segunda etapa donde se presentan deformaciones en el tiempo provocadas solo por el fenómeno de creep. 


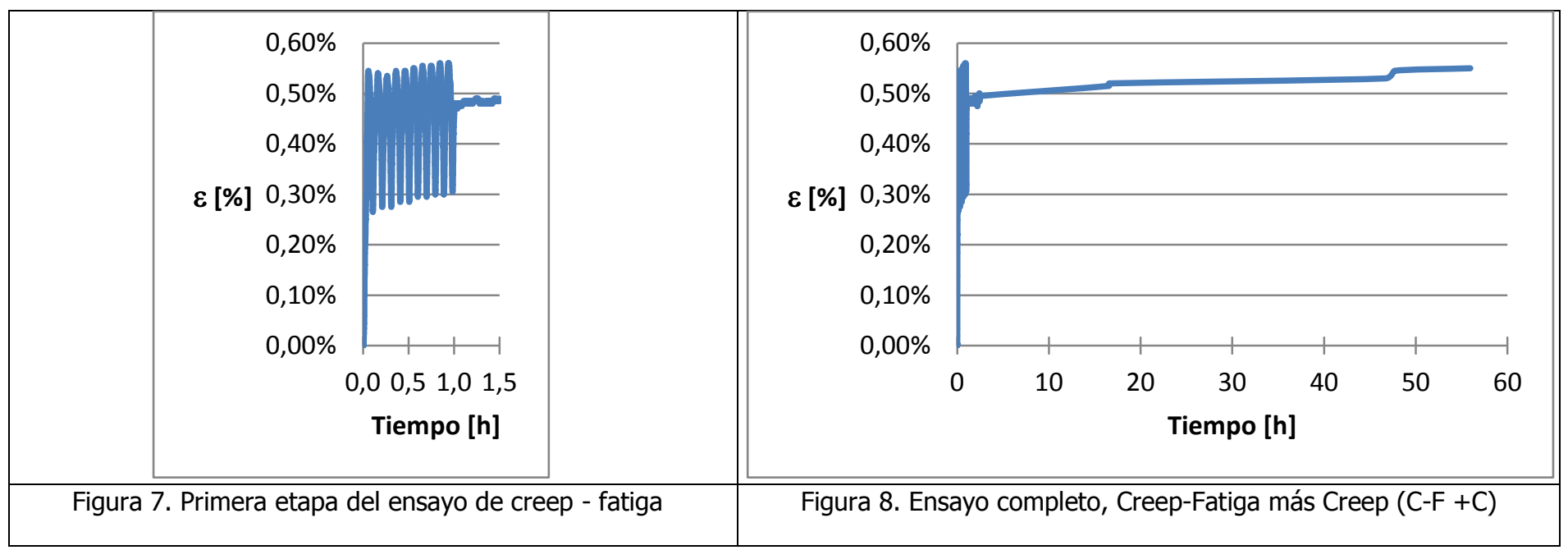

\section{Conclusiones}

Se logró visualizar claramente los fenómenos de creep y fatiga, y se observa la disminución de la vida del material cuando se somete a ciclos alternados de tensiones; este fenómeno se verificó en los dos grupos de ensayos que que se realizaron a tensiones máximas de 168 y 131 MPa respectivamente. También se justificó el comportamiento del material, a partir de las observaciones de las microestructuras.

Si bien no se expone por razones de espacio, se verifico a través de micrografías, la inexistencia de cambios micro estructurales tales como la globulización que permitan relacionarlos con el ablandamiento, lo cual es coherente con el corto tiempo de ensayo. Descartada esta opción y verificado el ablandamiento, se estima que el ablandamiento por fatiga podría explicarse por la generación de defectos puntuales que deja parcialmente el metal, permitiendo el trepado de dislocaciones fuera del plano de deslizamiento. Además, los ciclos de fatiga, particularmente los primeros, modifican significativamente la velocidad mínima de creep y en consecuencia, la vida útil del componente.

A partir de los estudios realizados y de los resultados que se muestran se observó que las deformaciones de ratcheting aumentan la velocidad mínima de creep, lo que implica una reducción de la vida útil del material.

Este trabajo de tesis se continuará estudiando el comportamiento del acero ferrítico con $5 \%$ de cromo, para relacionar la influencia de la composición química con el efecto de perdida de resistencia provocado por la interacción creep - fatiga.

\section{Referencias}

ASTM E139-11. (2011). Standard Test Method for Conducting Creep, Creep-Rupture, and Stress-Rupture Tests of Metallic Materials. (A. Internacional, Ed.) PA, USA. Obtenido de www.astm.org

ASTM E2714-13. (2013). Standard Test Method for Creep-Fatigue Testing. PA, USA: ASTM Internacional.

Dieter, G. E. (1988). Mechanical Metallurgy. (U. o. Maryland, Ed.) McGraw-Hill Book Company. doi:https://doi.org/10.1002/crat.2170230211

Halford, G. R. (1997). Heat-Resistant Materials (Specialty Handbook ed.). ASM Internacional.

Holdsworth, S. R. (2011). Component Assessment Data Requirements from Creep-Fatigue Tests. PA, USA: ASTM Internacional. doi:https://doi.org/10.1520/STP49932S

Molina, R., Pender, G., Gonzalez, G., \& Moro, L. (2018). Checking of the uniformity of results of simultaneous testing simultaneous creep. Matéria, 26(2). doi:https://doi.org/10.1590/S1517-707620180002.0358

Viswanathan, R. (1989). Damage Mechanisms and Life Assessment of High Temperature Components. Park Ohio, USA: ASM International.

Zhang, K., \& Jarir, A. (2018). Ratcheting behavior of Eurofer 97 at $550{ }^{\circ} \mathrm{C}$. Nuclear Materials and Energy.

doi:https://doi.org/10.1016/j.nme.2018.03.003 